\title{
Ji Son Jang
}

\section{El ustedeo en tres zonas del departamento de Antioquia (Colombia)*}

\section{The use of usted in three areas of the department of Antioquia (Colombia)}

Resumen: En este trabajo se presentan algunos resultados acerca del uso del usted de una investigación sobre la selección pronominal llevada a cabo en tres municipios del departamento colombiano de Antioquia: 1) La ciudad de Medellín, que se considera zona urbana; 2) Carmen de Viboral, que se comprende como una zona semi rural (a una hora de la ciudad en automóvil) y 3) Andes, que se entiende como una zona rural (se sitúa a unas tres horas de la ciudad en coche). Más concretamente, se muestra, por un lado, en cuál de las tres zonas se emplearía con más frecuencia el usted y, por el otro, en qué zona se observa más notablemente la dependencia entre la selección del usted y la edad del interlocutor. La población estudiada está constituida por informantes de edades entre los 13 y los 34 años. La variable social para este artículo es el lugar de la vivienda de los informantes, es decir, zona urbana (Medellín), semi rural (Carmen de Viboral) o rural (Andes) lo cual se ha correlacionado con la edad de los informantes. Para llevar a cabo esta investigación se aplicó un cuestionario, donde aparecen distintos tipos interaccionales, a los informantes de las zonas mencionadas, obteniéndose un total de 1.490 informantes. Tres técnicas estadísticas fueron aplicadas a estos datos: 1) tamaño de muestra óptimo para distribuciones multinomiales; 2) análisis de varianza con un solo factor (ANOVA); 3) prueba de hipótesis para proporciones. La estadística muestra que, por un lado, en la gran mayoría de relaciones interpersonales, en Andes se usa más el usted que en Carmen de Viboral y, asimismo, en este último municipio se emplea más el usted que en Medellín; por otro lado, en Medellín, en comparación con Carmen de Viboral o Andes, se usa más el usted con interlocutores de mayor edad que con los de la misma o menor edad. En otras palabras, en las zonas rurales se usa mucho más el usted que en la zona urbana, independientemente de la edad de los interlocutores, como marca de costumbres más conservadoras.

\footnotetext{
* Este artículo se deriva de la investigación "Fórmulas de tratamiento pronominales en Antioquia (Colombia): Primera Fase” financiada por el CODI de la Universidad de Antioquia (Número de acta: CODI 580 del 23 de julio de 2010).
} 
Palabras claves: formas de tratamiento pronominales, sociolingüística, Antioquia (Colombia), zona rural/urbana

Abstract: In this paper, we present some results of a research about the pronominal use of usted in three municipalities of the Colombian department of Antioquia: 1) The city of Medellin, considered to be an urban zone, 2) Carmen de Viboral, a semi rural area an hour from Medellin by car, and 3) Andes, a rural area located about three hours from Medellin by car. Respondents aged between 13 and 34 years old constitute the studied population. The social variable for this article is the place of the housing of the informants; that is to say, an urban area (Medellin), a semi rural zone (Carmen de Viboral) and a rural zone (Andes). A questionnaire was used for this research, listing different types of interaction in the mentioned areas and administered to 1490 informants. Three statistical techniques were applied to the data: 1) estimated optimal sample size for polynomial distributions, 2) analysis of variance (ANOVA), and 3) hypothesis test for proportions. The statistics shows that in the great majority of interpersonal relations in Andes usted is more used than in Carmen de Viboral and likewise in the latter municipality usted is more employed than in Medellin.

Keywords: address forms, sociolinguistics, Antioquia (Colombia), rural/urban zone

Ji Son Jang: Universidad de Antioquia, E-Mail: tetsuji_miyahara@comunicaciones.udea.net.co

\section{Introducción}

El uso de las fórmulas de tratamiento en el mundo hispanohablante se ha ido transformando de acuerdo con la situación geográfica, política o socioeconómica de cada región (Carrera y Álvarez, 2004, p. 227; Navarro, 2004, p. 216). Su uso como estrategia de cortesía fue mencionado por Brown y Levinson (1987, p. 107, pp. 179-181) ${ }^{1} \mathrm{y}$, asimismo, varios investigadores del español han señala-

1 Según estos autores, el tú y el vos se consideran como una de las estrategias de cortesía positiva o de solidaridad y el usted como una de las estrategias de cortesía negativa o de distanciamiento. Varios estudios han señalado que, aunque haya diferencia regional del aspecto pragmático de los pronombres tú/vos y usted, por lo general, el tú/vos se asocia con el concepto de solidaridad y el usted con el de no-solidaridad (Pedroviejo, 2004, p. 246; Serrano, 2000, 
do la importancia de adquirir un buen manejo de los pronombres personales para expresar lingüísticamente cortesía o descortesía (Boretti y Rigatuso, 2004, p. 145; Braun, 1988, p. 46; Bravo, 2001, p. 304; Guerra, 2008, p. 228; Iglesias, 2001, p. 256; Placencia, 2010, p. 344). Por el contrario puede generar una situación conflictiva incluso entre interlocutores nativos de la misma lengua (Blas, 2005, p. 19; Bolívar, 2001, p. 68; Hummel, 2010a, p 109; Páez, 1981, p. 49). Desde esta perspectiva, se han realizado numerosos estudios acerca de las fórmulas de tratamiento en español (Blas, 1994-95, 1995, 2003; Carrera y Álvarez, 2004; Medina, 2010a; Móccero, 2003, p. 346; Navarro, 2004; Serrano, 2000, p. 204). En el departamento colombiano de Antioquia - considerado como centro del vo$\mathrm{seo}^{2}$ en Colombia -, donde coexisten tres formas pronominales: tú, vos y usted, desde el trabajo de Montes (1967) no se habían realizado suficientes investigaciones de carácter empírico que se incluyeran datos actualizados sobre el empleo de estas formas de tratamiento.

Con el ánimo de llenar esta laguna, y desde un punto de vista socio-pragmático, hemos indagado en nuestros trabajos anteriores al presente este fenómeno en distintos tipos de interacción en la ciudad de Medellín, por parte de la población joven universitaria tomando como variables el estrato socioeconómi$\mathrm{Co}^{3}$, el sexo (Jang, 2010), la edad media y el origen urbano o rural de los informantes y de sus padres (Jang, 2012). En este último estudio, se ha observado que los informantes de origen rural con padres del mismo origen tienden a usar más el usted que el tú/vos en la mayoría de las relaciones interpersonales. Sin embargo, en ese estudio se ha trabajado únicamente con la población medellinense, mientras que en el actual se trabaja con la población de las tres zonas. En Jang (2012, p. 158) se formula la siguiente hipótesis: los habitantes de la ciudad de Medellín usarían más el tú/vos que el usted en comparación con los habitantes de la zona rural antioqueña. La comprobación de esta hipótesis es lo que nos proponemos presentar aquí.

p. 204). A este respecto, vale la pena mencionar el trabajo de Brown y Gilman (1960), considerado como pionero, en el cual se menciona el concepto de poder y solidaridad para la selección pronominal.

$2 \mathrm{El}$ voseo moderno es el tratamiento familiar empleado para referirse a la segunda persona del singular. Existen algunas regiones hispanoamericanas donde el voseo se emplea a través del pronombre tú con el voseo verbal, por ejemplo, en la capital uruguaya (Steffen, 2010, p. 454) o la chilena (Uber, 2010, p. 1053). El voseo medellinense, no obstante, siempre se realiza a través del pronombre vos con el voseo verbal (Jang, 2009).

3 En la sociedad colombiana está oficialmente establecido el sistema de estratos socioeconómicos que se dividen en seis grados: uno es el más bajo y seis es el más alto por el sector de la vivienda. En función del estrato varía por ejemplo, el valor de la vivienda, de servicios públicos y de los impuestos. Por esta razón, los que tienen mayor ingreso suelen vivir en los estratos altos. 
En el presente artículo se presentan algunos resultados acerca del uso del usted de acuerdo con una investigación sobre la selección pronominal llevada a cabo en tres municipios antioqueños: 1) la ciudad de Medellín ${ }^{4}$, que se considera zona urbana; 2) Carmen de Viboral, que se comprende como una zona semirural que queda a una hora de la ciudad en automóvil y 3) Andes, que se entiende como una zona rural que se sitúa a unas tres horas en coche de la ciudad. Más concretamente, los objetivos de este artículo son mostrar, por un lado, en cuál de las zonas antes mencionadas se emplearía con más frecuencia el usted, es decir, se trata de corroborar la hipótesis planteada en el trabajo anterior (Jang, 2012, p. 158) y, por el otro, en qué zona se observa más notablemente la dependencia entre la selección del usted y la edad del interlocutor. El resultado de análisis sobre la relación entre la selección pronominal y el estrato socioeconómico y el sexo de los informantes o de los interlocutores no se presenta en este artículo, ya que apenas está en el proceso de análisis. Si bien la variable sexo y el nivel socioeconómico han sido considerados no se correlacionan en este estudio con la distribución por zonas del uso pronominal. La población estudiada está constituida por informantes estudiantiles de edades entre los 13 y 34 años. La variable social para este artículo es el lugar de la vivienda de los informantes, es decir, zona urbana (Medellín), semi rural (Carmen de Viboral) o rural (Andes). En cuanto al origen de la población estudiada, se trabajó solamente con informantes de origen antioqueño cuyos padres también son del departamento de Antioquia, con el fin de evitar cualquier influencia de otros usos pronominales de otras regiones, las cuales están fuera del objetivo de este trabajo.

\section{Aspectos sociales en las fórmulas de tratamiento en español}

\subsection{La sociedad moderna y el predominio del tratamiento solidario}

Hasta hace algunas décadas, el predominio del tratamiento de distanciamiento sobre el de solidaridad era la corriente general en el mundo hispanohablante. Actualmente, sin embargo, en las sociedades modernas parece prevalecer el tratamiento de solidaridad sobre el de distanciamiento. Así lo afirman, entre otros

4 Capital del departamento de Antioquia, incluyendo su área metropolitana. 
autores, Areiza y García (2003, p.74), Blas (2003, p.681), Brown y Gilman (1960), Medina (2005, p. 100) y Molina (2002, p. 98). Esta tendencia se observaba ya en la década de los setenta (Lastra, 1972, p. 213). Al respecto, Pedroviejo (2004, p. 247) explica:

El progreso que el empleo del tú ha experimentado en la mayoría de las comunidades de habla hispánica podría ser analizado como un reflejo de las sociedades modernas y democráticas, donde cada vez se van limando los prejuicios y jerarquizaciones sociales, lo que ha contribuido a un crecimiento del uso de tú como forma de tratamiento adecuado, incluso cortés, en situaciones cada vez más numerosas.

Esta tendencia se ha observado en diferentes variedades del español a ambos lados del Atlántico (Blas, 1994-95, p. 24)5. En la península ibérica de siglos o decenios anteriores, en que la sociedad estaba muy jerarquizada, se empleaba el usted hacia los interlocutores que ocupaban posiciones sociales superiores, siendo también general ustedear a los interlocutores con quienes se tenía confianza (Calderón, 2001, p. 29-32; Molina, 2002, p. 101). Pero ya en la década del sesenta o setenta comenzaba a predominar el tuteo sobre el usted en las poblaciones jóvenes peninsulares. El usted del español peninsular conllevaba un significado pragmático de bastante distanciamiento (Moreno, 1986, pp.93-96). En los años noventa, parece que el tuteo recíproco gana mucho terreno sobre el usted en la sociedad peninsular (Molina, 1993, p. 262). Hoy día, en las zonas urbanas españolas, el uso de usted al dirigirse a los padres o a los abuelos se comprende como arcaizante o rural (Molina, 2002, p. 114) y, según Blas (2005, p. 13), predomina el tuteo en las relaciones familiares o entre amigos jóvenes ${ }^{6}$. Este último investigador apunta que en España, en el transcurso del último decenio, se aumentó el número de los universitarios que tutean a sus profesores desde el principio, aunque el porcentaje de los que utilizan el usted sigue siendo alto (Blas, 2005, p.16). Por último, en el español de Canarias igualmente se observa el predominio del tú sobre el usted (Medina, 2010b, p. 230). De lo descrito anteriormente se deduce que en la comunidad peninsular actual predomina fuertemente el tratamiento de solidaridad sobre el de distanciamiento ${ }^{7}$; y este hecho refleja que en la actualidad España es una sociedad más bien moderna.

5 Como un caso excepcional, sin embargo, Blas (2003, p. 672) señala que en la comunidad valenciana, se prefiere usar el usted por parte de los estudiantes universitarios al dirigirse a los profesores.

6 De acuerdo con Molina (2010, p. 876), en Madrid el tuteo se emplea con todos los miembros familiares independientemente de su edad o grado de parentesco.

7 A este respecto, véanse también Reid y Comajoan (2005). 
En cuanto a la selección pronominal en México, Lastra (1972, p. 215) apunta la misma tendencia predominante del tratamiento solidario sobre el de distanciamiento. Recientemente, Reid y Comajoan (2005) también la señalan.

De acuerdo con Rigatuso (1988-1989, pp. 89-90), en el español bonaerense de mediados del siglo XIX predominaba el uso de usted sobre el de los pronombres solidarios. Pero en el español bonaerense contemporáneo se observa el avance del voseo (tratamiento de solidaridad) hasta entre interlocutores desconocidos y esto se podría comprender como un fenómeno igualitario (Boretti, 2001, p. 83; Fontanella, 1970, p. 17, 21). En Argentina, la tendencia en favor del voseo entre desconocidos en puestos de atención al público está generalizada en la actualidad, ya sea en oficinas o comercios (Ferrer, 2003, p. 324; Sánchez, 2003, p. 337-338). Se acepta socialmente este voseo argentino generalizado entre interlocutores jóvenes y en creciente afianzamiento entre emisores de distinto nivel generacional (Ferrer, 2003, pp.330-331). En conclusión, en el español bonaerense actual las fórmulas solidarias están ganando terreno y se puede observar el uso de vos hasta entre desconocidos (Rigatuso, 2000, pp. 317-318) ${ }^{8}$.

Para el caso de Chile, Hummel (2010a, pp.105, 134) señala que se observa el avance del pronombre solidario sobre el de distanciamiento.

En lo que se refiere a la selección pronominal en República Dominicana, Alba (2004, p.146), citado por Hummel (2010b, p.300), afirma el avance del tuteo sobre el ustedeo, en comparación con décadas anteriores, en el trato de los hijos hacia sus padres o abuelos, incluso en las zonas rurales.

Según Hummel, Alzola (1969, p. 218) apunta que en Cuba el tuteo empezó a predominar sobre el ustedeo tempranamente, a partir de la época colonial (Hummel (2010b, p. 300); igualmente, Callejas (1983, p. 111) señala la progresión del tuteo sobre el ustedeo en Santiago de Cuba (Hummel (2010b, p. 302).

Álvarez y Freites (2010, p. 333) dicen que en el estudio de los pronombres en Mérida (Venezuela) de Álvarez y New (2003) se observa el debilitamiento del tratamiento distanciador, aunque su ritmo es lento.

En lo que se refiere a la selección pronominal bogotana en contextos comerciales, Placencia apunta que: "Uber (1997) describe [...] que la solidaridad está cobrando la mayor importancia que el poder [...].” (Placencia, 2010, p. 349).

Para el caso ecuatoriano, Placencia apunta que: “Toscano Mateus (1953) describe el uso de cuatro pronombres: usted, tú, vos y su merced [sumercé]. [...] En cuanto a su empleo, nota, por ejemplo, que dentro de la familia en zonas

8 En cuanto al avance del tratamiento solidario sobre el de distanciamiento en Argentina, Carricaburo (2010, p. 477) afirma que en la época de Eva Duarte de Perón se produjo un rápido avance en el eje de la solidaridad propiciado desde el gobierno central y cuya influencia alcanzó las distintas zonas dialectales argentinas. 
urbanas, los hijos se dirigen a sus padres de usted, pero que el uso de tú está en aumento" (Placencia, 2010, p. 353).

Según Hummel (2010c, p. 391), en el trabajo de Sologuren (1954, pp. 247248) se observa que en Lima ya desde el siglo XVIII mediaba el indicio del predominio del tuteo sobre el tratamiento distanciador.

Para el caso de la capital uruguaya, Steffen (2010) señala:

Behares (1981: 33) nota en la misma ciudad "una apertura de las relaciones sociales hacia formas solidarias de tratamiento". Del mismo modo, los datos del ADDU evidencian un avance de las formas de confianza en el tratamiento de los hijos al dirigirse a los padres (Mendoza 2005: 110-125) (Steffen, 2010, p. 459).

Como se ha expuesto en las páginas anteriores, en diferentes variedades hispanohablantes se observa el avance del tratamiento solidario sobre el de distanciamiento. Este fenómeno parece ser mucho más notable en la sociedad ibérica, sobre todo en la madrileña, en comparación con algunas regiones americanas de habla hispana, por ejemplo, con la mexicana (Reid y Comajoan, 2005; Schwenter, 1993, citados por Vázquez y Orozco, 2010, p. 259), la chilena (Hummel, 2010a, pp.105, 134), la costarricense (Hummel, 2010c, p. 396) o la uruguaya (Steffen, 2010, p. 459).

\subsection{Correlaciones conservadoras o innovadoras en función de la zona urbana/rural}

En el apartado anterior se ha señalado que en el español de hoy en día predomina el tratamiento solidario sobre el de distanciamiento. En cuanto a este aspecto, por lo general, las zonas urbanas propician y acogen las innovaciones lingüísticas, mientras que las rurales tienden a ser conservadoras (Moreno, 1992, p. 350, nota 16). En lo concerniente a las fórmulas de tratamiento, Álvarez y Carrera señalan que: “[...] según Fontanella (1999), en las comunidades más conservadoras, especialmente en las rurales, perduran usos con mayor predominio de las relaciones asimétricas, mientras que en las más innovadoras habría un avance de las formas solidarias y cercanas” (Álvarez y Carrera, 2006, p. 123). Molina, en su estudio sobre la selección pronominal de los jóvenes madrileños, afirma:

Los datos muestran cómo en la comparación de Madrid capital con otros lugares de procedencia la ciudad siempre presenta el sello de innovadora. Otros estudios del castellano peninsular (Borrego Nieto et al. 1978, Aguado Candanedo 1980) confirman que la novedad comienza por las ciudades, centros de población desde los que irradia el cambio social "saltando" de un centro urbano a otro, y de ahí se extiende a las comunidades rurales. Así por ejemplo, hay restos de usos arcaizantes - como el empleo del pronombre usted 
para el trato con los padres o con los abuelos - que los hablantes identifican como propios del ámbito rural. Este uso está hoy estigmatizado en el medio urbano como arcaizante y rural (Molina, 2002, p.114).

En el español de Canarias también se observa que el ustedeo hacia los padres, en comparación con el tuteo, se arraiga en las zonas rurales (Medina, 2010b, pp. 230-231).

En la investigación sobre la evolución pronominal bonaerense, Fontanella dice que el predominio del concepto de solidaridad sobre el de distanciamiento se generó en la población urbana, ya que esta es más abierta a cambios en los usos y costumbres; la población rural, en cambio, es más apegada a la norma anterior (Fontanella, 1970, p. 17) ${ }^{9}$.

Kluge, para el caso de Chile, señala: "Para Santiago, se postula una tendencia entre los jóvenes a utilizar el tuteo [...], apartándose del uso de usted en muchas ocasiones en las cuales sus compañeros en el sur de Chile sí lo emplean” (Kluge, 2005, p. 187).

Igualmente, Hummel (2010a, p. 117), con base en Eguiluz (1962), afirma que el ustedeo chileno en el ámbito familiar, en comparación con el tuteo, tenía una imagen rural ya en la década de 1960 .

Para el caso del uso pronominal en las ciudades mexicanas (Ciudad de México y Guadalajara), Vázquez y Orozco (2010, pp. 258-259) señalan:

[...] la extensión del tuteo es un fenómeno que se propaga de las grandes ciudades a las ciudades pequeñas o a las áreas rurales, pues la frecuencia de tuteo es mayor entre los nacidos en la ciudad de Guadalajara que entre personas de pequeños poblados (Orozco 2006), y los hablantes cuyos padres son originarios de Guadalajara tutean más que aquellos cuyos padres provienen de áreas rurales.

De lo descrito en las páginas anteriores, se infiere que en la zona urbana se puede destacar la característica lingüística innovadora, es decir, el predominio del tú/vos y, en cambio, en la zona rural permanece el carácter lingüístico conservador, o lo que es lo mismo, el arraigo del usted. En lo que se refiere a este aspecto, por último, merece la pena mencionar que Moreno, en su estudio acerca de la selección pronominal en una comunidad rural española (Quintanar de la Orden, provincia de Toledo), afirma que tanto mujeres como hombres tratan siempre de tú a los amigos o familiares jóvenes (Moreno, 1986, p.114) y señala que el ustedeo entre jóvenes quintanareños es absolutamente minoritario (Moreno, 1986, p.117). Lo apuntado por este autor es bastante interesante, ya que

9 Para el caso de formas pronominales bonaerenses del siglo XIX, esta misma investigadora afirma que "en cuanto al habla rural, presenta a principios del siglo XIX un uso más conservador que el de los hablantes urbanos, [...]” (Fontanella, 1985, p. 21). 
implica que el tú en España predominaba sobre el usted incluso en una comunidad rural desde la década del ochenta.

\section{Corpus y tratamiento estadísticos de datos}

\subsection{La naturaleza de datos}

El corpus principal del presente estudio se fundamenta en los datos obtenidos a través de las encuestas aplicadas a los informantes en las tres zonas mencionadas $^{10}$. Como variables sociales analizadas en este artículo se considera el lugar de vivienda de los informantes (zona urbana, semi rural y rural), y, debido a la dificultad de conseguir informantes de los estratos altos en las zonas semi-rurales y rurales, hemos decidido trabajar solo con los informantes de los estratos populares, es decir, 1, 2 y 3. La gran mayoría de los cuestionarios se aplicaron en las instituciones universitarias o en los colegios $^{11}$ de dichas zonas. En el cuestionario se preguntó a los informantes con qué pronombre (tú, vos o usted) tratarían a diferentes interlocutores, los cuales fueren elegidos teniendo en cuenta cómo variaría la selección de cada forma de tratamiento, principalmente dependiendo del rol relativo que el informante tenga con respecto a cada interlocutor. Los interlocutores que aparecen en el cuestionario son como muestra la siguiente tabla $1^{12}$ :

Tabla 1: Interlocutores que aparecen en el cuestionario

\begin{tabular}{ll}
\hline Pregunta & ¿Cómo trata (o trataría) usted a las siguientes personas? \\
\hline 1 & La madre \\
2 & El padre \\
3 & La hermana mayor \\
4 & La hermana menor \\
5 & El hermano mayor \\
6 & El hermano menor \\
7 & La abuela \\
\hline
\end{tabular}

10 Los datos de la presente investigación se recogieron entre septiembre de 2010 y marzo de 2011. Al final del presente estudio, también se muestran algunos ejemplos de las grabaciones de las conversaciones reales.

11 Todos los informantes son estudiantes universitarios o del grado octavo, noveno, décimo y once del colegio bachillerato que es un ciclo de estudios anterior a la educación universitaria en Colombia.

12 Los numerales que aparecen en la tabla 1 corresponden a los números de las preguntas del cuestionario. 
Tabla 1: continuación

\begin{tabular}{ll}
\hline Pregunta & ¿Cómo trata (o trataría) usted a las siguientes personas? \\
\hline 8 & El abuelo \\
9 & La hija de 20 años \\
10 & La hija de 5 años \\
11 & El hijo de 20 años \\
12 & El hijo de 5 años \\
13 & El/La novio/novia (en privado) \\
14 & El/La novio/novia (ante amigos) \\
15 & El/La novio/novia (ante los padres) \\
16 & El/La novio/novia (ante desconocidos) \\
17 & El/La esposo/esposa o compañero/compañera \\
18 & Una amiga 10 años mayor \\
19 & Una amiga de la misma edad \\
20 & Un amigo 10 años mayor \\
21 & Un amigo de la misma edad \\
22 & Una señora de 60 años que no se conoce \\
23 & Una joven de 20 años que no se conoce \\
24 & Un señor de 60 años que no se conoce \\
25 & Un joven de 20 años que no se conoce \\
26 & Una niña de 5 años \\
27 & Un niño de cinco años \\
28 & Una profesora muy amiga de 60 años \\
29 & Una profesora muy amiga de 30 años \\
30 & Un profesor muy amigo de 60 años \\
31 & Un profesor muy amigo de 30 años \\
32 & Una profesora de 60 años con la que no tiene confianza \\
33 & Una profesora de 30 años con la que no tiene confianza \\
34 & Un profesor de 60 años con el que no tiene confianza \\
35 & Una médica de 60 años en su consultorio \\
36 & Un médico de 60 años en su consultorio \\
37 & \\
38 & Un médico de 30 años en su consultorio \\
39 & Un de 30 años \\
40 & añosa \\
\hline 1 &
\end{tabular}

\subsection{Tratamiento estadístico de datos}

En esta investigación se apeló a cuatro técnicas estadísticas: 1) tamaño de muestra óptimo para distribuciones multinomiales (Thompson, 1987); 2) análisis de varianza con un solo factor (ANOVA); 3) prueba de hipótesis para proporciones. Este procesamiento se ha realizado con el programa $\mathrm{R}$. 


\subsubsection{Tamaño de muestra óptimo para distribuciones multinomiales}

El tamaño de la muestra tiene como objetivo seleccionar aleatoriamente el menor número de observaciones $n$ que represente a la población total (Thompson, 1987). Los tres parámetros definidos para la estimación del tamaño de la muestra fueron:

1. Nivel de confianza del $99 \%(1-\alpha$ con $\alpha=0,01)$

2. Precisión absoluta de $+5 \%(d=0,05)$

3. $m=3$ (las categorías para las fórmulas de tratamiento pronominales: tú, vos, usted)

\subsubsection{Análisis de varianza con un solo factor (ANOVA)}

Se apeló a la prueba del análisis de varianza con un solo factor (ANOVA) con el fin de verificar si la edad media en tres zonas son iguales o no, es decir, para determinar la influencia de la edad en el análisis de los datos. Si la prueba nos muestra que la edad media en las tres zonas es igual, podremos analizar sin preocuparnos por la influencia de ésta.

La hipótesis planteada es:

$\mathrm{H}_{0}: \mu_{1}=\mu_{2}=\mu_{3}$ (La edad media es igual en las tres zonas)

$\mathrm{H}_{\mathrm{i}}$ : No todas las $\mu_{\mathrm{g}}$ son iguales.

La hipótesis nula $\left(\mathrm{H}_{0}\right)$ no se acepta cuando el $p$-value es inferior a 0,05 y tiene un nivel de confianza del 95\%; en caso contrario, no se puede rechazar.

\subsubsection{Prueba de hipótesis para proporciones}

La prueba de hipótesis para proporciones fue empleada en esta investigación para establecer si existen diferencias significativas en la selección de usted en función de la variable el lugar de vivienda del informante.

Las hipótesis planteadas son:

$\mathrm{H}_{0}: p_{1}=p_{2}=p_{3}$ (Las proporciones son iguales en tres zonas)

$\mathrm{H}_{\mathrm{i}}$ : No todas las $p_{\mathrm{g}}$ son iguales.

La hipótesis nula se rechaza si el valor de la significancia asintótica (Asymp. Sig.) es menor que 0,05, permitiendo afirmar que existen diferencias significativas entre las tres zonas analizadas. De esta manera, se pretendió observar, por un lado, en qué zona se usaría más el usted y, por el otro, en qué zona depen- 
dería la selección del usted en función de la edad del interlocutor, por ejemplo, si se emplearía más el usted con una hermana mayor que con una hermana menor.

\section{Análisis}

\subsection{Introducción}

Con base en el tamaño de muestra óptimo para distribuciones multinomiales, el tamaño de muestra $n$ estimado fue de 765 personas y, aplicando encuestas a 4.207 personas, se consiguieron 1.490 cuestionarios válidos. Con ello, se obtuvo una cantidad suficiente de informantes para un análisis corroborado estadísticamente. Véase la tabla 2 para los detalles de los informantes:

Tabla 2: Distribución de informantes

\begin{tabular}{llrrrr}
\hline \multirow{2}{*}{$\begin{array}{l}\text { Lugar de vivienda del } \\
\text { informante }\end{array}$} & Sexo & \multicolumn{2}{c}{ Estrato socioeconómico } & \multirow{2}{*}{ Total } \\
\cline { 2 - 5 } & & $\mathbf{1}$ & $\mathbf{2}$ & $\mathbf{3}$ & \\
\hline Área Metropolitana & Femenino & 45 & 256 & 188 & $\mathbf{4 8 9}$ \\
& Masculino & 42 & 187 & 164 & 393 \\
\hline Carmen de Viboral & Femenino & 2 & 68 & 106 & $\mathbf{1 7 6}$ \\
\hline Andes & Masculino & 2 & 25 & 45 & $\mathbf{7 2}$ \\
\hline Total & Femenino & 32 & 146 & 37 & $\mathbf{2 1 5}$ \\
\hline
\end{tabular}

Según la prueba del análisis de varianza con un solo factor (ANOVA), la edad media en tres zonas es estadísticamente uniforme (Valor P: 0,804), es decir, la diferencia de la edad en función de la zona no afecta a nuestro análisis. Esto

Tabla 3: La edad media en tres zonas

\begin{tabular}{lllll}
\hline $\begin{array}{l}\text { Edad de los } \\
\text { informantes }\end{array}$ & \multicolumn{3}{c}{ Lugar de vivienda } & \multirow{2}{*}{ General } \\
\cline { 2 - 4 } & Área Metropolitana & Carmen de Viboral & Andes & \\
\hline Edad mínima & 14 & 14 & 13 & 13 \\
Edad máxima & 34 & 32 & 34 & 34 \\
Edad media & 20 & 16 & 17 & 19 \\
\hline
\end{tabular}


nos permite realizar un análisis riguroso solo con la variable el lugar de vivienda del informante. Véase la tabla 3:

\subsection{Frecuencia del usted en tres municipios antioqueños}

De acuerdo con la tabla 4, la estadística muestra que, en la gran mayoría de relaciones interpersonales, en Andes (zona rural) se usa más el usted que en Carmen de Viboral (zona semi rural) y, asimismo, en este último municipio se emplea más el usted que en Medellín (zona urbana). Es decir, en la zona rural se emplea más el usted que en la zona semi rural y en esta última más que en la zona urbana.

Tabla 4: Prueba de hipótesis para proporciones del usted ${ }^{13}$

\begin{tabular}{|c|c|c|c|c|}
\hline \multirow[t]{3}{*}{ Preguntas } & \multicolumn{3}{|c|}{ Lugar de vivienda del informante } & \multirow{2}{*}{$\begin{array}{l}\text { Igualdad de } \\
\text { proporciones }\end{array}$} \\
\hline & Area Metropolitana & Carmen de Viboral & Andes & \\
\hline & Usted & Usted & Usted & Sig. \\
\hline 1 & $50,60 \%$ & $71,40 \%$ & $73,10 \%$ & $0,000^{\star}$ \\
\hline 2 & $56,00 \%$ & $73,80 \%$ & $75,50 \%$ & $0,000^{*}$ \\
\hline 3 & $39,80 \%$ & $69,20 \%$ & $67,50 \%$ & $0,000^{*}$ \\
\hline 4 & $36,50 \%$ & $68,80 \%$ & $70,80 \%$ & $0,000^{*}$ \\
\hline 5 & $37,60 \%$ & $69,50 \%$ & $71,80 \%$ & $0,000^{\star}$ \\
\hline 6 & $36,70 \%$ & $67,20 \%$ & $71,70 \%$ & $0,000^{*}$ \\
\hline 7 & $59,30 \%$ & $75,80 \%$ & $76,00 \%$ & $0,000^{*}$ \\
\hline 8 & $60,50 \%$ & $77,20 \%$ & $74,80 \%$ & $0,000^{*}$ \\
\hline 9 & $33,00 \%$ & $51,60 \%$ & $56,60 \%$ & $0,000^{\star}$ \\
\hline 10 & $26,20 \%$ & $50,60 \%$ & $53,20 \%$ & $0,000^{*}$ \\
\hline 11 & $30,60 \%$ & $55,60 \%$ & $58,40 \%$ & $0,000^{*}$ \\
\hline 12 & $26,20 \%$ & $50,60 \%$ & $53,20 \%$ & $0,000^{*}$ \\
\hline 13 & $23,30 \%$ & $32,80 \%$ & $34,10 \%$ & $0,000^{*}$ \\
\hline 14 & $30,40 \%$ & $48,80 \%$ & $50,60 \%$ & $0,000^{*}$ \\
\hline 15 & $34,00 \%$ & $53,40 \%$ & $55,80 \%$ & $0,000^{*}$ \\
\hline 16 & $30,70 \%$ & $46,20 \%$ & $49,20 \%$ & $0,000^{*}$ \\
\hline 17 & $25,60 \%$ & $43,90 \%$ & $48,60 \%$ & $0,000^{*}$ \\
\hline 18 & $46,00 \%$ & $63,30 \%$ & $65,80 \%$ & $0,000^{*}$ \\
\hline 19 & $30,40 \%$ & $56,90 \%$ & $57,80 \%$ & $0,000^{*}$ \\
\hline 20 & $47,80 \%$ & $62,50 \%$ & $68,20 \%$ & $0,000^{*}$ \\
\hline 21 & $34,80 \%$ & $62,80 \%$ & $63,10 \%$ & $0,000^{*}$ \\
\hline 22 & $81,00 \%$ & $85,90 \%$ & $85,00 \%$ & 0,089 \\
\hline
\end{tabular}

13 En la última columna se presentan valores de la significancia asintótica y los que son estadísticamente significativos (menores a 0,05$)$ se marcan con un asterisco ${ }^{\star}{ }^{*}$. 
Tabla 4: continuación

\begin{tabular}{lllll}
\hline \multirow{2}{*}{ Preguntas } & \multicolumn{2}{l}{ Lugar de vivienda del informante } & Igualdad de \\
\cline { 2 - 3 } & Area Metropolitana & Carmen de Viboral & Andes & \\
\cline { 2 - 4 } & Usted & Usted & Usted & Sig. \\
\hline 23 & $70,10 \%$ & $74,20 \%$ & $77,70 \%$ & $0,019^{\star}$ \\
24 & $79,60 \%$ & $85,10 \%$ & $86,70 \%$ & $0,006^{\star}$ \\
25 & $70,30 \%$ & $71,00 \%$ & $83,50 \%$ & $0,000^{\star}$ \\
26 & $37,00 \%$ & $48,80 \%$ & $63,60 \%$ & $0,000^{\star}$ \\
27 & $36,50 \%$ & $48,80 \%$ & $63,10 \%$ & $0,000^{\star}$ \\
28 & $58,30 \%$ & $68,50 \%$ & $69,00 \%$ & $0,000^{\star}$ \\
29 & $53,30 \%$ & $66,50 \%$ & $68,30 \%$ & $0,000^{\star}$ \\
30 & $58,30 \%$ & $72,60 \%$ & $73,80 \%$ & $0,000^{\star}$ \\
31 & $54,00 \%$ & $70,40 \%$ & $73,30 \%$ & $0,000^{\star}$ \\
32 & $80,30 \%$ & $83,50 \%$ & $82,10 \%$ & 0,465 \\
33 & $79,00 \%$ & $83,10 \%$ & $83,60 \%$ & 0,106 \\
34 & $81,60 \%$ & $83,50 \%$ & $83,80 \%$ & 0,586 \\
35 & $80,80 \%$ & $81,50 \%$ & $86,40 \%$ & 0,066 \\
36 & $81,30 \%$ & $79,40 \%$ & $80,70 \%$ & 0,812 \\
37 & $77,90 \%$ & $75,80 \%$ & $78,80 \%$ & 0,681 \\
38 & $81,20 \%$ & $81,50 \%$ & $83,10 \%$ & 0,737 \\
39 & $79,60 \%$ & $79,40 \%$ & $82,50 \%$ & 0,473 \\
40 & $82,20 \%$ & $83,50 \%$ & $85,00 \%$ & 0,493 \\
41 & $82,20 \%$ & $84,30 \%$ & $82,80 \%$ & 0,746 \\
\hline
\end{tabular}

Como se ha visto en las páginas anteriores, la tendencia actual en las fórmulas de tratamiento pronominales es la preferencia del tratamiento solidario sobre el de distanciamiento (Blas, 2005, p.13; Boretti, 2001, p. 83; Calderón, 2001, p. 29-32; Ferrer, 2003, p. 324; Fontanella, 1970, p. 17; Lastra, 1972, p. 215; Molina, 1993, p. 262; 2002, p.101; Moreno, 1986, pp. 93-96; Rigatuso, 1988-1989, pp. 89-90; 2000, pp.317-318; Sánchez, 2003, pp.337-338). Por lo general, la población rural suele apegarse a la norma lingüística conservadora, es decir, el predominio del tratamiento distanciado sobre el solidario; en cambio, en las zonas urbanas prevalece la norma lingüística innovadora, en otras palabras, la predominancia del tú/vos sobre el usted (Álvarez y Carrera, 2006, p. 123; Fontanella, 1970, p.17; 1985, p. 21; Molina, 2002, p.114; Kluge, 2005, p. 187). El caso del presente estudio precisamente coincide con esta tendencia y en el estudio de Jang (2012) en la ciudad de Medellín se ha observado que los informantes de origen rural con padres del mismo origen tienden a emplear más el usted que el tú/vos en la mayoría de relaciones interpersonales. De esto se infiere que en el departamento de Antioquia, muy posiblemente, ha pasado lo mismo que se ha 
observado en otras regiones hispanohablantes: el paso del uso predominante del usted al del tú/vos a partir de la zona urbana.

Ahora bien, si tenemos en cuenta lo que señala, por ejemplo, Moreno (1986, p.117), en su investigación sobre los tratamientos en una comunidad rural española, en la que el autor afirma que en esta comunidad rural predominaba el tú sobre el usted desde la década del ochenta, se supone que no se observaría gran diferencia en las fórmulas de tratamiento pronominales peninsulares actuales en función de la zona urbano-rural. Pero el presente estudio sobre el uso pronominal muestra una diferencia notable en este aspecto. Considerando a Páez (1981) - quien muestra la historia de la evolución del uso pronominal, más concretamente, quien señala que donde y cuando había una sociedad igualitaria se simplificaba el sistema pronominal y se complicaba cuando y donde había una sociedad jerarquizada -, como se ha visto anteriormente, cabe afirmar que en el departamento de Antioqueña hay una gran diferencia en la norma de la selección pronominal entre la zona urbana y la rural, debido a que, por ejemplo, hay una notable distancia sociocultural y socioeconómica entre las dos zonas.

Por otro lado, cabe aclarar que, para tratar a los profesores con los que no se tiene confianza, a los médicos y a los sacerdotes, la estadística no muestra la diferencia del uso del usted en función de la zona ${ }^{14}$, es decir, los informantes de tres zonas los tratan de usted de una manera similar. Esto quiere decir que no solo en las zonas rurales, sino también en la zona urbana es general el uso del usted hacia los interlocutores desconocidos o que ejercen poder social. Asimismo, se debe destacar que, a pesar de que en la zona rural se emplea con más frecuencia el usted que en la zona urbana, incluso en la zona urbana (Medellín) se utiliza con bastante frecuencia hasta en las relaciones interpersonales solidarias (por ejemplo, la mitad de los informantes medellinenses tratan de usted a sus padres) y no como el caso de la capital peninsular donde el tuteo se emplea con todos los miembros familiares independientemente de su edad o grado de parentesco (Molina, 2010, p. 876).

\subsection{Dependencia entre la selección del usted y la edad del interlocutor en tres municipios antioqueños}

De acuerdo con las tablas 5, 6 y 7, la estadística muestra que la dependencia entre la selección del usted y la edad del interlocutor se observa más notable-

14 Ver los resultados de las preguntas 32 - 41 de la tabla 4. Todos los datos estadísticos indican inferiores a 0,05 para estas preguntas. 
mente en Medellín (zona urbana) que en Carmen de Viboral (zona semi rural) o Andes (zona rural); es decir, en Medellín, en comparación con Carmen de Viboral o Andes, se usa más el usted al referirse a los interlocutores de mayor edad que a los oyentes de la misma edad o menor edad. De hecho, en más de la mitad de los casos de las relaciones interpersonales en Medellín se observa esta tendencia, pero en Carmen de Viboral o Andes, prácticamente no se observa esta tendencia ${ }^{15}$.

Tabla 5: Prueba de hipótesis para proporciones de la dependencia entre la selección del usted y la edad del interlocutor: el caso de Medellín ${ }^{16}$

\begin{tabular}{|c|c|c|c|c|c|c|}
\hline \multirow[t]{3}{*}{ Preguntas } & \multirow{2}{*}{\multicolumn{2}{|c|}{$\frac{\text { Informante Femenino }}{\text { Interlocutores }}$}} & \multicolumn{4}{|c|}{ Informante Masculino } \\
\hline & & & \multirow{2}{*}{$\begin{array}{l}\text { Sig. } \\
\text { Asintótica }\end{array}$} & \multicolumn{2}{|c|}{ Interlocutores } & \multirow{2}{*}{$\begin{array}{l}\text { Sig. } \\
\text { Asintótica }\end{array}$} \\
\hline & Mayor & Menor & & Mayor & Menor & \\
\hline 3 Vs 4 & $38,00 \%$ & $36,50 \%$ & 0,418 & $41,70 \%$ & $36,10 \%$ & $0,004^{*}$ \\
\hline 5 Vs 6 & $37,80 \%$ & $36,80 \%$ & 0,575 & $37,10 \%$ & $36,60 \%$ & 0,888 \\
\hline 9 Vs 10 & $29,60 \%$ & $22,80 \%$ & $0,000^{*}$ & $37,10 \%$ & $30,50 \%$ & $0,001^{*}$ \\
\hline 11 Vs 12 & $26,70 \%$ & $23,50 \%$ & 0,054 & $35,10 \%$ & $30,60 \%$ & $0,028^{\star}$ \\
\hline 18 Vs 19 & $45,30 \%$ & $30,90 \%$ & $0,000^{*}$ & $46,80 \%$ & $29,80 \%$ & $0,000^{*}$ \\
\hline 20 Vs 21 & $45,30 \%$ & $30,90 \%$ & $0,000^{*}$ & $46,80 \%$ & $29,80 \%$ & $0,000^{*}$ \\
\hline 22 Vs 23 & $82,30 \%$ & $73,30 \%$ & $0,000^{*}$ & $79,40 \%$ & $65,90 \%$ & $0,000^{*}$ \\
\hline 24 Vs 25 & $81,80 \%$ & $72,30 \%$ & $0,000^{*}$ & $77,00 \%$ & $67,90 \%$ & $0,000^{*}$ \\
\hline 28 Vs 29 & $60,90 \%$ & $55,10 \%$ & $0,000^{\star}$ & $55,20 \%$ & $50,90 \%$ & $0,010^{*}$ \\
\hline 30 Vs 31 & $61,80 \%$ & $57,90 \%$ & $0,013^{\star}$ & $54,10 \%$ & $49,20 \%$ & $0,006^{*}$ \\
\hline 32 Vs 33 & $82,00 \%$ & $81,40 \%$ & 0,663 & $78,30 \%$ & $76,00 \%$ & 0,110 \\
\hline 34 Vs 35 & $85,30 \%$ & $84,00 \%$ & 0,286 & $77,10 \%$ & $76,80 \%$ & 1,000 \\
\hline 36 vs 37 & $81,70 \%$ & $79,90 \%$ & 0,137 & $80,70 \%$ & $75,30 \%$ & $0,000^{*}$ \\
\hline 38 Vs 39 & $82,00 \%$ & $80,00 \%$ & 0,100 & $80,20 \%$ & $79,10 \%$ & 0,556 \\
\hline 40 vs 41 & $83,60 \%$ & $84,50 \%$ & 0,480 & $80,40 \%$ & $79,40 \%$ & 0,423 \\
\hline
\end{tabular}

15 Según las tablas, esta tendencia se observa en Medellín en siete (7) de quince (15) casos en total para los informantes femeninos y diez (10) de quince (15) casos en total para los informantes masculinos. En cambio, en Carmen de Viboral se observa esta tendencia apenas en dos (2) de (15) casos en total para los informantes de ambos sexos. Asimismo, en Andes se observa esta tendencia solo en tres (3) de quince (15) casos en total para los informantes femeninos y uno (1) de quince (15) casos en total para los informantes masculinos.

16 En la primera columna, por ejemplo, estamos examinado la frecuencia del uso del usted comparando la pregunta 3 (tratamiento para una hermana mayor) con la 4 (tratamiento para una hermana menor). Como se ha aclarado anteriormente el sexo está fuera del análisis para este artículo. 
Tabla 6: Prueba de hipótesis para proporciones de la dependencia entre la selección del usted y la edad del interlocutor: el caso de Carmen de Viboral

\begin{tabular}{|c|c|c|c|c|c|c|}
\hline \multirow[t]{3}{*}{ Preguntas } & \multirow{2}{*}{\multicolumn{2}{|c|}{$\frac{\text { Informante Femenino }}{\text { Interlocutores }}$}} & \multicolumn{4}{|c|}{ Informante Masculino } \\
\hline & & & \multirow{2}{*}{$\begin{array}{l}\text { Sig. } \\
\text { Asintótica }\end{array}$} & \multicolumn{2}{|c|}{ Interlocutores } & \multirow{2}{*}{$\begin{array}{l}\text { Sig. } \\
\text { Asintótica }\end{array}$} \\
\hline & Mayor & Menor & & Mayor & Menor & \\
\hline 3 Vs 4 & $72,70 \%$ & $69,30 \%$ & 0,114 & $60,60 \%$ & $67,60 \%$ & 0,131 \\
\hline 5 Vs 6 & $72,60 \%$ & $70,30 \%$ & 0,386 & $62,00 \%$ & $60,60 \%$ & 1,000 \\
\hline 9 Vs 10 & $56,10 \%$ & $53,80 \%$ & 0,502 & $41,40 \%$ & $42,90 \%$ & 1,000 \\
\hline 11 Vs 12 & $56,60 \%$ & $52,60 \%$ & 0,121 & $52,90 \%$ & $44,30 \%$ & 0,077 \\
\hline 18 Vs 19 & $61,90 \%$ & $59,10 \%$ & 0,560 & $66,70 \%$ & $51,40 \%$ & $0,015^{\star}$ \\
\hline 20 Vs 21 & $61,10 \%$ & $61,70 \%$ & 1,000 & $66,70 \%$ & $65,30 \%$ & 1,000 \\
\hline 22 Vs 23 & $85,80 \%$ & $72,20 \%$ & $0,000^{*}$ & $86,10 \%$ & $79,20 \%$ & 0,228 \\
\hline 24 Vs 25 & $84,10 \%$ & $69,30 \%$ & $0,000^{*}$ & $87,50 \%$ & $75,00 \%$ & $0,039^{*}$ \\
\hline 28 Vs 29 & $69,90 \%$ & $68,80 \%$ & 0,752 & $65,30 \%$ & $61,10 \%$ & 0,546 \\
\hline 30 Vs 31 & $74,90 \%$ & $72,60 \%$ & 0,289 & $66,70 \%$ & $65,30 \%$ & 1,000 \\
\hline 32 Vs 33 & $85,20 \%$ & $83,00 \%$ & 0,289 & $79,20 \%$ & $83,30 \%$ & 0,450 \\
\hline 34 Vs 35 & $83,50 \%$ & $81,80 \%$ & 0,371 & $83,30 \%$ & $80,60 \%$ & 0,617 \\
\hline 36 vs 37 & $79,00 \%$ & $76,70 \%$ & 0,453 & $80,60 \%$ & $73,60 \%$ & 0,228 \\
\hline 38 Vs 39 & $80,10 \%$ & $77,80 \%$ & 0,343 & $84,70 \%$ & $83,30 \%$ & 1,000 \\
\hline 40 vs 41 & $83,50 \%$ & $86,40 \%$ & 0,131 & $83,30 \%$ & $79,20 \%$ & 0,450 \\
\hline
\end{tabular}

Tabla 7: Prueba de hipótesis para proporciones de la dependencia entre la selección del usted y la edad del interlocutor: el caso de Andes

\begin{tabular}{|c|c|c|c|c|c|c|}
\hline \multirow[t]{3}{*}{ Preguntas } & \multirow{2}{*}{\multicolumn{2}{|c|}{$\frac{\text { Informante Femenino }}{\text { Interlocutores }}$}} & \multicolumn{4}{|c|}{ Informante Masculino } \\
\hline & & & \multirow{2}{*}{$\begin{array}{l}\text { Sig. } \\
\text { Asintótica }\end{array}$} & \multicolumn{2}{|c|}{ Interlocutores } & \multirow{2}{*}{$\begin{array}{l}\text { Sig. } \\
\text { Asintótica }\end{array}$} \\
\hline & Mayor & Menor & & Mayor & Menor & \\
\hline 3 Vs 4 & $70,30 \%$ & $73,60 \%$ & 0,190 & $64,50 \%$ & $66,70 \%$ & 0,628 \\
\hline 5 Vs 6 & $74,40 \%$ & $75,80 \%$ & 0,579 & $68,10 \%$ & $65,20 \%$ & 0,386 \\
\hline 9 Vs 10 & $58,90 \%$ & $56,00 \%$ & 0,239 & $52,60 \%$ & $49,60 \%$ & 0,453 \\
\hline 11 Vs 12 & $61,50 \%$ & $57,20 \%$ & $0,039^{*}$ & $53,30 \%$ & $53,30 \%$ & 1,000 \\
\hline 18 Vs 19 & $68,80 \%$ & $60,00 \%$ & $0,017^{\star}$ & $61,40 \%$ & $54,50 \%$ & 0,175 \\
\hline 20 Vs 21 & $70,00 \%$ & $62,00 \%$ & $0,015^{\star}$ & $65,70 \%$ & $65,70 \%$ & 1,000 \\
\hline 22 Vs 23 & $88,80 \%$ & $85,00 \%$ & 0,118 & $79,30 \%$ & $66,90 \%$ & $0,003^{*}$ \\
\hline 24 Vs 25 & $93,00 \%$ & $89,70 \%$ & 0,169 & $77,60 \%$ & $74,10 \%$ & 0,359 \\
\hline 28 Vs 29 & $71,00 \%$ & $72,00 \%$ & 0,773 & $66,00 \%$ & $63,90 \%$ & 0,628 \\
\hline 30 Vs 31 & $75,70 \%$ & $76,20 \%$ & 1,000 & $71,00 \%$ & $69,70 \%$ & 0,803 \\
\hline 32 Vs 33 & $88,30 \%$ & $89,30 \%$ & 0,724 & $72,90 \%$ & $75,00 \%$ & 0,450 \\
\hline 34 Vs 35 & $88,40 \%$ & $89,80 \%$ & 0,450 & $77,60 \%$ & $81,10 \%$ & 0,131 \\
\hline 36 vs 37 & $83,20 \%$ & $82,70 \%$ & 1,000 & $78,00 \%$ & $72,30 \%$ & 0,080 \\
\hline 38 Vs 39 & $85,10 \%$ & $84,70 \%$ & 1,000 & $80,00 \%$ & $79,30 \%$ & 1,000 \\
\hline 40 vs 41 & $88,40 \%$ & $87,40 \%$ & 0,724 & $79,90 \%$ & $76,40 \%$ & 0,228 \\
\hline
\end{tabular}


De este resultado y de lo descrito en el apartado anterior, se infiere que el usted se usa mucho en las zonas rurales tanto para referirse a los interlocutores mayores como para tratar a los de la misma edad o edad menor, como una marca del arraigo de la preferencia del tratamiento conservador. Y, a nuestro juicio, este resultado no necesariamente quiere decir que se respete más a los mayores en la zona urbana que en las zonas rurales.

\section{Consideraciones finales}

En este trabajo que, como se ha comentado anteriormente, es parte de una investigación mayor, se ha trabajado con una población limitada estudiantil y el margen de edad es relativamente reducido. Por otra parte variables que pueden ser significativas, se tratan de un modo solo marginal, como el sexo y el estrato socioeconómico de los informantes. Sin embargo, de lo descrito a lo largo del presente trabajo, en resumen, se puede afirmar que en el departamento colombiano de Antioquia, como se observa en otras regiones hispanohablantes, muy posiblemente antes fuera la corriente general el predominio del usted sobre el tú/vos en todas las zonas, pero que ahora existiría una notable diferencia dependiendo de si la zona es urbana, semi rural o rural, distribución que estaría marcando un cambio en el uso. Es decir, con base en los datos del presente estudio, la tendencia pronominal anterior muy probablemente se ha cambiado paulatinamente por la preferencia del tú/vos desde la zona urbana y, por eso, en el departamento estudiado se emplea más el usted en la zona rural que en la zona semi rural y en esta última más que en la zona urbana. Y, debido a esto, en Medellín (zona urbana), en comparación con Carmen de Viboral (zona semi rural) o Andes (zona rural) se usa más el usted al referirse a los interlocutores de mayor edad que a los oyentes de la misma edad o menor edad, en otras palabras, en las zonas rurales se usa mucho más el usted que en la zona urbana, independiente a la edad de los interlocutores, como una marca de la costumbre conservadora.

En el marco de la noción de la cortesía, se puede señalar que en cada sociedad y en cada época se observa la tendencia del predominio de una norma y algunas estrategias de las dos caras de la cortesía: la solidaria o la distanciadora. A este respecto, se puede afirmar que, en Antioquia predomina la cortesía de distanciamiento (usted) en la zona rural y la cortesía de solidaridad (tú/vos) en la zona urbana. Para corroborar lo dicho con más rigurosidad, se requiere realizar un estudio diacrónico que hasta el momento no existe. 


\section{Referencias}

Álvarez, A. y Carrera, M. (2006). El usted de solidaridad en el habla de Mérida. En M. SchraderKniffki (ed.), La cortesía en el mundo hispánico. Nuevos contextos, nuevos enfoques metodológicos (pp. 117-130). Frankfurt: Vervuert Iberoamericana.

Álvarez, A. y Freites, F. (2010). Los estudios sobre pronombres de segunda persona en Venezuela. En M. Hummel et al., (eds.), Formas y fórmulas de tratamiento en el mundo hispánico (pp. 325-339). México DF: Colegio de México.

Areiza, R. y García, A. (2003). ¿Qué significa saludar? En D. Bravo (ed.), Actas del Primer Coloquio del programa Edice: "La perspectiva no etnocentrista de la cortesía: identidad sociocultural de las comunidades hispanohablantes” (pp. 71-85). Estocolmo: Universidad de Estocolmo.

Blas, J. (1994-95). Tú y usted: dos pronombres de cortesía en el español actual. Datos de una comunidad peninsular. Estudios de Lingüística, 10, 21-44.

Blas, J. (1995). Los pronombres de tratamiento y la cortesía. Revista de Estudios Hispánicos (Universidad de Puerto Rico), 22, 439-466.

Blas, J. (2003). Perspectivas (socio)lingüísticas complementarias en el estudio de la variación y el cambio lingüístico en español. Estudios de Sociolingüística, 4(2), 653-692.

Blas, J. (2005). Los grados de la cortesía verbal: reflexiones en torno a algunas estrategias comunicativas y recursos lingüísticos en el español peninsular contemporáneo. Revista Internacional de Lingüística Iberoamericana, volumen III, 1(5), 9-29.

Bolívar, A. (2001). El insulto como estrategia en el diálogo político venezolano. Oralia, 4, 47-73.

Boretti, S. (2001). Aspectos de la cortesía lingüística en el español coloquial de Argentina. Oralia, 4, 75-102.

Boretti, S. y Rigatuso, E. (2004). La investigación de la cortesía en el español de la Argentina. Estado de la cuestión. En D. Bravo et al., (eds.), Pragmática sociocultural: estudios sobre el discurso de cortesía en español (pp. 137-167). Barcelona: Ariel.

Braun, F. (1988). Terms of address. Problems of usage in various languages and cultures. Berlin: Mouton de Gruyter.

Bravo, D. (2001). Sobre la cortesía lingüística, estratégica y conversacional en español. Oralia, 4, 299-314.

Briz, A. (2003). La estrategia atenuadora en la conversación cotidiana española. En D. Bravo (ed.), Actas del primer coloquio del programa Edice: "La perspectiva no etnocentrista de la cortesía: identidad sociocultural de las comunidades hispanohablantes" (pp.17-46). Estocolmo: Universidad de Estocolmo.

Brown, R. y Gilman, A. (1960). The pronouns of Power and Solidality. En T. A. Sebeok (ed.), Style in Language (pp. 253-276), Cambridge/Mass: MIT Press.

Brown, P., y Levinson S. (1987). Politeness. Some universals in language usage. Cambridge: Cambridge University Press.

Calderón, M. (2001). Fórmulas de tratamientos sociales en obras literarias Andaluzas de la segunda mitad del siglo XIX. Cuadernos del Sur, 31, 7-35.

Carrera, M. y Álvarez, A. (2004). Tratamientos y cortesía en la elaboración de fuentes documentales de la etapa fundacional de la provincia de Mérida (Venezuela). En D. Bravo et al., (eds.), Pragmática sociocultural: estudios sobre el discurso de cortesía en español (pp. 227-243). Barcelona: Ariel. 
Carricaburo, N. (2010). Estudios fundantes del voseo en la Argentina. En M. Hummel et al., (eds.), Formas y fórmulas de tratamiento en el mundo hispánico (pp. 465-481). México DF: Colegio de México.

Ferrer, M. (2003). El discurso de la cortesía en puestos de atención al público en Argentina. En D. Bravo (ed.), Actas del Primer Coloquio del programa Edice: "La perspectiva no etnocentrista de la cortesía: identidad sociocultural de las comunidades hispanohablantes" (pp. 315-331). Estocolmo: Universidad de Estocolmo.

Fontanella, M. (1970). La evolución de los pronombres de tratamiento en el español bonaerense. Tesaurus, 25(1), 12-22.

Fontanella, M. (1985). La evolución del voseo bonaerense en el siglo XIX. Estudios Filológicos, 20, $9-24$.

Guerra, N. (2008). El discurso conflictivo en interacciones coloquiales en inglés y español. El uso de las fórmulas de tratamiento. En A. Briz et al., (eds.), Cortesía y conversación: de lo escrito a lo oral, III Coloquio Internacional del Programa EDICE (pp. 224-239). Valencia/Estocolmo: Universidad de Valencia y Programa EDICE.

Hummel, M. (2010a). Reflexiones metodológicas y teóricas sobre el estudio de las formas de tratamiento en el mundo hispanohablante, a partir de una investigación en Santiago de Chile. En M. Hummel et al., (eds.), Formas y fórmulas de tratamiento en el mundo hispánico (pp.101-162). México DF: Colegio de México.

Hummel, M. (2010b). El estudio de las formas de tratamiento en las Antillas hispanohablantes. En M. Hummel et al., (eds.), Formas y fórmulas de tratamiento en el mundo hispánico (pp. 293-323). México DF: Colegio de México.

Hummel, M. (2010c). El estudio de las formas de tratamiento en Perú. En M. Hummel et al., (eds.), Formas y fórmulas de tratamiento en el mundo hispánico (pp.375-398). México DF: Colegio de México.

Iglesias, S. (2001). Los estudios de la cortesía en el mundo hispánico. Estado de la cuestión. Oralia, 4, 245-298.

Jang, J. (2009). Fórmulas de tratamiento pronominales en los jóvenes universitarios de Medellín: una aproximación socio-pragmática. Tesis doctoral. Kansai Gaidai University.

Jang, J. (2010). Fórmulas de tratamiento pronominales en los jóvenes universitarios de Medellín (Colombia) desde la óptica socio-pragmática: estrato socioeconómico y sexo. Íkala, 15(26), 43-116.

Jang, J. (2012). Correlaciones entre la selección pronominal, el origen urbano / rural y la edad: el caso de jóvenes universitarios de Medellín (Colombia). Íkala, 17(2), 145-166.

Kluge, B. (2005). Las fórmulas de tratamiento en un corpus chileno. En V. Noll et al., (eds.), El español en América: Aspectos teóricos, particularidades, contactos (pp.169-188). Frankfurt: Vervuert Iberoamericana.

Lastra, Y. (1972). Los pronombres de tratamiento en la ciudad de México. Anuario de Letras, 10, 213-217.

Medina, J. (2005). Variación sociolingüística en las formas de trato. El análisis probabilístico según los datos del español de Canarias. En J. M. Medrano (ed.), Actas del II Coloquio Internacional del Programa EDICE: "Actos de habla y cortesía en distintas variedades del español: Perspectivas teóricas y metodológicas” (pp.97-114). Costa Rica/Estocolmo: Universidad de Costa Rica y Programa EDICE.

Medina, J. (2010a). El tuteo presidencial: ¿descortesía o cambio de "talante"? En F. Orletti et al., (eds.), (Des)cortesía en español. Espacios teóricos y metodológicos para su estudio (pp. 209-220). Roma-Estocolmo: Università degli Studi Roma Tre-Edice. 
Medina, J. (2010b). Panorama sobre el estudio de las formas de tratamiento en el español de Canarias. En M. Hummel et al., (eds.), Formas y fórmulas de tratamiento en el mundo hispánico (pp. 223-246). México DF: Colegio de México.

Móccero, M. (2003). Las selecciones pronominales como estrategias de cortesía. En D. Bravo (ed.), Actas del Primer Coloquio del programa EDICE: "La perspectiva no etnocentrista de la cortesía: identidad sociocultural de las comunidades hispanohablantes" (pp. 346-354). Estocolmo: Universidad de Estocolmo.

Molina, I. (1993). Las fórmulas de tratamiento de los jóvenes madrileños. Estudio sociolingüístico. LEA, 15(2), 249-263.

Molina, I. (2002). Evolución de las fórmulas de tratamiento en la juventud madrileña a lo largo del siglo XX: un estudio en tiempo real. En F. Rodríguez (coord.), El lenguaje de los jóvenes (pp. 97-121). Barcelona : Ariel.

Molina, I. (2010). Inmigración latinoamericana en Madrid: actitudes lingüísticas y convergencia pronominal. En M. Hummel et al., (eds.), Formas y fórmulas de tratamiento en el mundo hispánico (pp. 857-885). México DF: Colegio de México.

Montes, J. (1967). Sobre el voseo en Colombia. Thesaurus, XXII, 1, 21-44.

Moreno, F. (1986). Sociolingüística de los tratamientos. Estudio sobre una comunidad rural. Anuario de Letras, 24, 87-120.

Moreno, F. (1992). Norma y prestigio en el español de América. Apuntes para una planificación de la lengua española. Revista de Filología Española, 72(3/4), 345-359.

Navarro, R. (2004). Formas De cortesía en la Segunda Celestina. En D. Bravo (eds.), Pragmática sociocultural: estudios sobre el discurso de cortesía en español (pp. 213-225). Barcelona: Ariel.

Páez, I. (1981). Historia y geografía hispanoamericana del voseo. Caracas: La casa de Bello.

Pedroviejo, J. (2004). Formas de tratamientos en dos obras de teatro del siglo XX: Historia de una escalera y Bajarse al moro. En D. Bravo (eds.), Pragmática sociocultural: estudios sobre el discurso de cortesía en español (pp. 245-262). Barcelona: Ariel.

Placencia, M. (2010). El estudio de formas de tratamiento en Colombia y Ecuador. En M. Hummel et al., (eds.), Formas y fórmulas de tratamiento en el mundo hispánico (pp. 341-373). México DF: Colegio de México.

Reid, L. y Comajoan, L. (2005). El uso actual de tú y usted en el español peninsular y mexicano. Ponencia presentada en el VLLL Congreso Nacional de Lingüística, Asociación Mexicana de Lingüística Aplicada/Universidad de las Américas, Pue., 17 al 20 de mayo de 2005, Cholula, Pue.

Rigatuso, E. (1988-1989). Fórmulas de tratamiento sociales en el español bonaerense de mediados del siglo XIX. Cuadernos del Sur, 21/22, 65-93.

Rigatuso, E. (2000). Señora (...) ¿No tenés más chico? Un aspecto de la pragmática de las fórmulas de tratamiento en español bonaerense. Revista Argentina de Lingüística, 16, 293-344.

Sánchez, C. (2003). El discurso de la cortesía en puestos de atención al público en la Argentina (instituciones de salud pública y bienestar social). En D. Bravo (ed.), Actas del Primer Coloquio del programa EDICE: "La perspectiva no etnocentrista de la cortesía: identidad sociocultural de las comunidades hispanohablantes” (pp. 332-345). Estocolmo: Universidad de Estocolmo.

Serrano, M. (2000). La producción de la cortesía verbal y la deixis socio-comunicativa. Oralia, 3, 199-219. 
Steffen, J. (2010). El tratamiento en Uruguay. En M. Hummel et al., (eds.), Formas y fórmulas de tratamiento en el mundo hispánico (pp.449-464). México DF: Colegio de México.

Thompson, S. (1987). Sample Size for Estimating Proportions. The American Statistician, 41, (1), 42-46.

Uber, D. (2010). Formas y fórmulas de trato en situaciones laborales en Santiago de Chile y Buenos Aires. En M. Hummel et al., (eds.), Formas y fórmulas de tratamiento en el mundo hispánico (pp.1051-1080). México DF: Colegio de México.

Vázquez, M. y Orozco, L. (2010). Formas de trato del español en México. En M. Hummel et al., (eds.), Formas y fórmulas de tratamiento en el mundo hispánico (pp. 247-269). México DF: Colegio de México.

Ji Son Jang es Doctor en Estudios de Lenguaje y Cultura de la Universidad de Kansai Gaidai, Japón. Actualmente es profesor de tiempo completo de la Universidad de Antioquia, Colombia.

Ji Son Jang holds a $\mathrm{PhD}$ in Language and Culture Studies from Kansai Gaidai University, Japan. He currently works as full time professor at Universidad de Antioquia, Colombia.

\section{Apéndice}

A continuación se muestran algunos ejemplos reales ${ }^{17}$. En el ejemplo (1), interactúan dos hermanas, habitantes de Andes (zona rural). Diana es estudiante de bachillerato y tiene 16 años. Juana es universitaria y tiene 19 años. Las dos se tratan de usted:

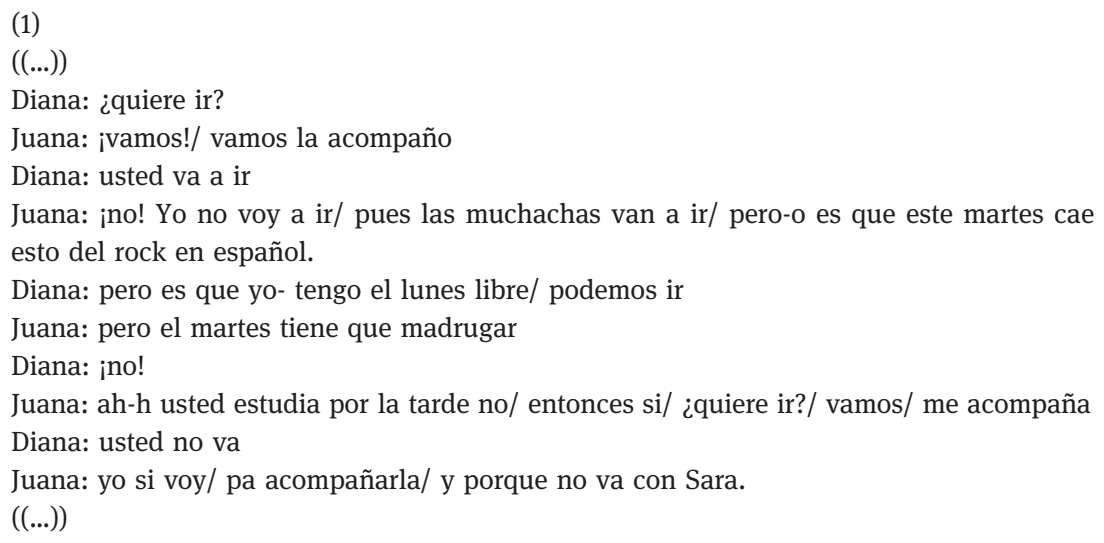

17 Los nombres propios que se mencionan en los ejemplos son ficticios. Las transcripciones se hicieron con base en las convenciones sistematizadas por el grupo Val.Es.Co; véase, por ejemplo, Briz (2003, pp. 45-46). 
En el ejemplo (2), interactúan dos hermanas, habitantes de Carmen de Viboral (zona semi rural). María es universitaria y tiene 22 años. Lina trabaja y tiene 30 años. Las dos se tratan de vos:

(2)

((...))

María: Claro que mirá que hay algo muy curioso, aquí laboralmente hay más trabajo para las mujeres que para los hombres

Lina: ¿En serio, gorda?

María: Aquí hay mucho más empleo para las mujeres que para los hombres

Lina: Y ¿por qué vos supiste por lo que estás estudiando?

$((\ldots))$

En el ejemplo (3), por último, interactúan dos hermanas, habitantes de Medellín (zona urbana). Luisa es universitaria y tiene 24 años. Sofía trabaja y tiene 26 años. Luisa trata a su hermana mayor de usted y Sofía de vos a su hermana menor:

(3)

Luisa: Quiubo y ¿Cómo le fuee en la cita del odontólogo ¿Qué le dijeron?//

Sofía: Imaginate que me estaban/ mirando pues lo de la muela y esa muela está llena llena de una infección estaba por allá llena de comida entonces// imaginate que me echaroon unn ácido ahí ¡Ah! No un ácido no izque agua oxigenada//

Luisa: Jumm ¿Y eso sí le sirve?//

Sofía: Claaro eso sí me sirvió/ porque ahí mismo que me echaron eso eso me dolió mucho mucho//

$((\ldots))$ 